\title{
Upgrade of the CMS Global Muon Trigger
}

\author{
Manfred Jeitler, Joschka Lingemann, Dinyar Rabady, Hannes Sakulin, and Achim Stahl
}

\begin{abstract}
The increase in center-of-mass energy and luminosity for Run-II of the Large Hadron Collider poses new challenges for the trigger systems of the experiments. To keep triggering with a similar performance as in Run-I, the CMS muon trigger is currently being upgraded. The new algorithms will provide higher resolution, especially for the muon transverse momentum and will make use of isolation criteria that combine calorimeter with muon information already in the level-1 trigger. The demands of the new algorithms can only be met by upgrading the level-1 trigger system to new powerful FPGAs with high bandwidth I/O. The processing boards will be based on the new $\mu$ TCA standard. We report on the planned algorithms for the upgraded Global Muon Trigger $(\mu \mathrm{GMT})$ which sorts and removes duplicates from boundaries of the muon trigger sub-systems. Furthermore, it determines how isolated the muon candidates are based on calorimetric energy deposits. The $\mu$ GMT will be implemented using a processing board that features a large Xilinx Virtex 7 FPGA. Up to 72 optical links at $10 \mathrm{~Gb} / \mathrm{s}$ will be used to receive muon candidates and energy sums from the calorimeter trigger. Muon candidates will be received directly from the sector processors of the upgraded trigger, absorbing the final sorting stage of each muon sub-system and thus minimizing the latency of the trigger.
\end{abstract}

Index Terms-CMS, isolation, muon, trigger.

\section{INTRODUCTION}

$\mathbf{T}$ HE Large Hadron Collider (LHC) is in the process of being upgraded for its second running period, to start in 2015. The center-of-mass energy will be increased from $\sqrt{s}=8$ $\mathrm{TeV}$ to $13 \mathrm{TeV}$. In Run-I, the number of interactions per collision (pile-up) as well as the instantaneous luminosity surpassed the design parameters of 25 interactions per bunch crossing and $10^{34} \mathrm{~cm}^{-2} \mathrm{~s}^{-1}$, respectively. They will increase even further in Run-II to 50 interactions per bunch crossing and more than $2 \times 10^{34} \mathrm{~cm}^{-2} \mathrm{~s}^{-1}$. Overall, this leads to an increase of roughly a factor of six in the total expected event rate [1].

Manuscript received June 28, 2014; revised December 15, 2014; accepted May 17, 2015. Date of publication June 05, 2015; date of current version June $12,2015$.

M. Jeitler is with the Institut für Hochenergiephysik der OeAW, 1050 Wien, Austria.

J. Lingemann is with the European Organization for Nuclear Research, CERN, 1211 Geneva 23, Switzerland, and also with the 3 Physikalisches Institut B, RWTH Aachen, 52074 Aachen, Germany (e-mail: joschka.lingemann@cern.ch).

D. Rabady is with the European Organization for Nuclear Research, CERN, 1211 Geneva 23, Switzerland, and also with the Institut für Hochenergiephysik der OeAW, 1050 Wien, Austria.

H. Sakulin is with the European Organization for Nuclear Research, CERN, 1211 Geneva 23, Switzerland.

A. Stahl is with the 3 Physikalisches Institut B, RWTH Aachen, 52074 Aachen, Germany.

Color versions of one or more of the figures in this paper are available online at http://ieeexplore.ieee.org.

Digital Object Identifier 10.1109/TNS.2015.2435060

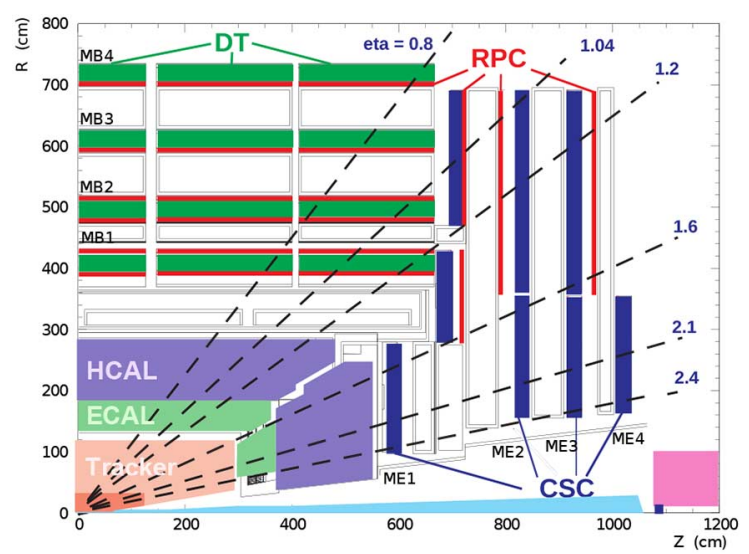

Fig. 1. One quadrant of the CMS detector. The four DT stations in the barrel (Muon Barrel, MB1-MB4), the four CSC stations in the end-cap (Muon Endcap, ME1-ME4) and the RPC stations are shown together with the tracker and calorimeter systems consisting of hadronic calorimeter (HCAL) and electromagnetic calorimeter (ECAL).

The Compact Muon Solenoid (CMS) experiment is one of the multi-purpose detectors at the LHC. A schematic view can be found in Fig. 1. Muons are reconstructed using the silicon tracker at the center of the detector that is immersed in the $3.8 \mathrm{~T}$ magnetic field, and the dedicated muon detector system interleaved with the steel return yoke. The total coverage of the muon system in pseudorapidity is $|\eta|<2.4^{1}$. The muon system consists of Drift Tubes (DT) in the barrel region with a coverage of $|\eta|<1.2$ and Cathode Strip Chambers (CSC) in the end-caps $(0.9<|\eta|<2.4)$. Both are complemented by Resistive Plate Chambers (RPC) covering the range of $|\eta|<1.6$. These acceptances define three detector regions with different instrumentation: The barrel $(|\eta|<0.8)$, the overlap $(0.8<|\eta|<1.2)$ and the end-cap $(\eta>1.2)$ region. The read-out frequency of the detector is limited to roughly $100 \mathrm{kHz}$ by the front-end electronics. To select events of interest, a two-level triggering scheme is applied. The level-1 trigger is hardware-based and reduces the rate from the LHC bunch crossing rate of $40 \mathrm{MHz}$ to $100 \mathrm{kHz}$. For this first decision only local information is processed at coarse granularity. The second High Level Trigger stage runs on a computer farm and combines the information in more sophisticated but slower algorithms. A more detailed description of the CMS detector may be found elsewhere [2].

For the broad physics program of the CMS experiment, which includes both searches for new physics at the TeV scale as well

\footnotetext{
${ }^{1} \mathrm{~A}$ right-handed coordinate system is used, with the origin at the nominal collision point. The $x$-axis points to the center of the LHC ring, the y-axis points up, and the $\mathrm{z}$ axis along the beam direction. The pseudorapdity $\eta$ is defined as $\eta=-\ln \tan (\theta / 2)$, where $\cos \theta=p_{z} / p$. The azimuthal angle $\phi$ is the angle to the $\mathrm{x}$-axis in the $\mathrm{x}-\mathrm{y}$ plane. The radius $r$ is the distance from the $\mathrm{z}$-axis.
} 
as measurements of Standard Model parameters, a high performance trigger system is crucial. This means that interesting physics processes have to be identified with a high efficiency while on the other hand the read-out rate has to be manageable. Muons, as minimum ionizing particles, are ideal candidates for triggering: While most particles are absorbed in the calorimeter system, the muons are also measured in the muon chambers. An introduction into typical methods of triggering in high-energy physics may be found elsewhere [3].

The true transverse momentum $\left(p_{T}\right)$ spectrum of muons falls steeply. However, the $p_{T}$ resolution at the level-1 trigger has tails. Muons of low $p_{T}$ can thus be promoted to higher $p_{T}$ at the level-1 trigger. This effect is called feed-through. The muon rate is therefore dominated by muons that actually have low $p_{T}$ but are promoted to high $p_{T}$. The integral spectrum levels out. Consequently, larger increases in the $p_{T}$ thresholds have to be accepted to significantly decrease the rate. Therefore, to keep thresholds low enough to have high efficiency for interesting physics processes while maintaining a manageable rate, the level-1 trigger system has to be upgraded and new algorithms have to be introduced. One such algorithm is the muon isolation that is motivated by the wide-spread use of such criteria in CMS analyses of processes with muons in the final state, where it is used to suppress events from background processes [4]. Muons can be produced in the actual collision or in the subsequent decay of other particles. In the latter case these other particles deposit energy in the calorimeters. Muons as minimum ionizing particles traverse the entire detector and leave signals in the muon chambers and tracker. The footprint of a muon in the calorimeter system is small. The isolation makes use of these facts and rejects muons where coincidences between large calorimeter deposits and the muon tracks can be established.

\section{A. The Current Muon Trigger System}

The current muon trigger chain is shown in Fig. 2. All three muon systems of CMS provide trigger information. In the barrel region, hits from the DT and in the end-caps from the CSC are combined to track segments. This information is then used in the local trigger, the DT and CSC Track Finders, to reconstruct full tracks and to assign physical properties. Hits from the RPC, which cover both barrel and end-cap regions, provide their own track candidates. These are reconstructed based on a pattern recognition algorithm [5]. Up to four candidates from CSC and DT track finders and up to four candidates each for barrel and end-cap RPCs are sent to the GMT. The candidate information includes the transverse momentum $p_{T},(\eta, \phi)$-coordinates, the charge and a quality code. In a first step, DT and CSC candidates are matched with the barrel and end-cap RPC candidates, based on the $\eta$ and $\phi$ coordinates. If a match is found, the candidates are merged and a corresponding $p_{T}$ is assigned. If two candidates can be merged, the lower of the $p_{T}$-measurements is assigned to the final candidate in most regions of the detector. This reduces the feed-through effect mentioned above, by reducing the number of muons that are promoted to a higher $p_{T}$.

The three muon systems overlap in acceptance. Duplicate muons from the overlap regions are canceled. Un-matched candidates can optionally be suppressed in the single-muon

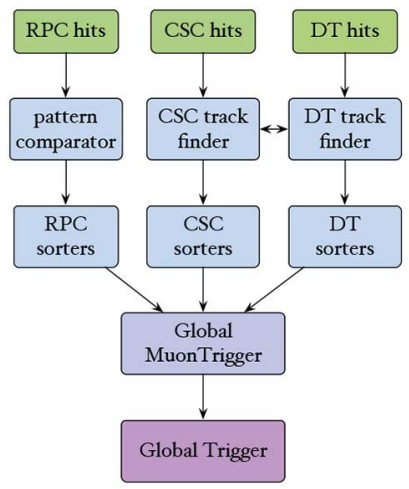

Fig. 2. The current muon trigger chain, from the muon sub-detector hits via the track finders to the GMT and to the final module, the GT.

trigger based on their quality and $\eta$. The final muon candidates are then sent to the Global Trigger (GT) which forms the final level-1 trigger decision. The current level-1 trigger system features mainly Field Programmable Gate Arrays (FPGA) and Application-Specific Integrated Circuits. Inter-card communication is realized mostly with galvanic parallel links. A more detailed description of the current level-1 trigger system may be found in [2] and a discussion of differences to and parallels with the ATLAS trigger may be found in [6].

\section{B. Motivation for an Upgraded Trigger System}

As mentioned previously, a significant increase in the eventrate is expected for 2015 and beyond. At the end of Run-I the level-1 trigger required single muons to have a $p_{T}$ of $16 \mathrm{GeV}$. Without modifications to the current triggering scheme, the $p_{T}$ thresholds for triggers requiring one muon would have to be as high as $42 \mathrm{GeV}$ [1]. This would significantly reduce the efficiency for measurements of processes containing, for example, a $\mathrm{W}$ boson. For di-muon triggers lower thresholds can be retained but current estimates still indicate problems for the selection of lighter resonances in the di-muon spectrum [1]. Therefore, existing algorithms have to be improved and additional information has to be used. The upgrade refines the $p_{T}$ measurement and adds the possibility to measure isolation.

Newly available more powerful FPGAs increase the logic resources significantly and allow for more complex algorithms to be implemented in the trigger. Combining the RPC and CSC (DT) information at an earlier stage can improve the $p_{T}$ assignment [1], directly reducing the triggering rate by minimizing the feed-through effect mentioned above. Requiring the muons to be isolated will help to decrease the rate even further. Moreover, the significant increase in input / output bandwidth in modern FPGAs allows a higher number of bits per variable as shown in Table I, reducing the loss of information through this discretization. Not only is the precision of the muon properties improved, but also the number of candidates can be higher throughout the trigger-chain. This bandwidth is possible through the utilization of high-speed serial links at up to $10 \mathrm{~Gb} / \mathrm{s}$ instead of parallel connections. These also simplify the system but will increase the latency due to the introduced (de)serialization stages.

The ATLAS collaboration also is in the process of upgrading its level-1 trigger in preparation for Run-II. In the ATLAS muon 
TABLE I

Number of Available Bits Per Property in Current and Upgraded GMT. THE DATA-FORMAT IS THE SAME FOR MUONS TRANSMITTED FROM THE Sub-Systems TO THE GMT AND FROM THE GMT TO THE GT

\begin{tabular}{l||c|c|c|c|c|c}
\hline variable & $p_{T}$ & $\phi$ & $\eta$ & quality & charge & calorimeter $E_{T}$ \\
\hline \hline current system & 5 & 8 & 6 & 3 & 2 & n/a \\
\hline upgraded system & 9 & 10 & 9 & 4 & 2 & 5 \\
\hline
\end{tabular}

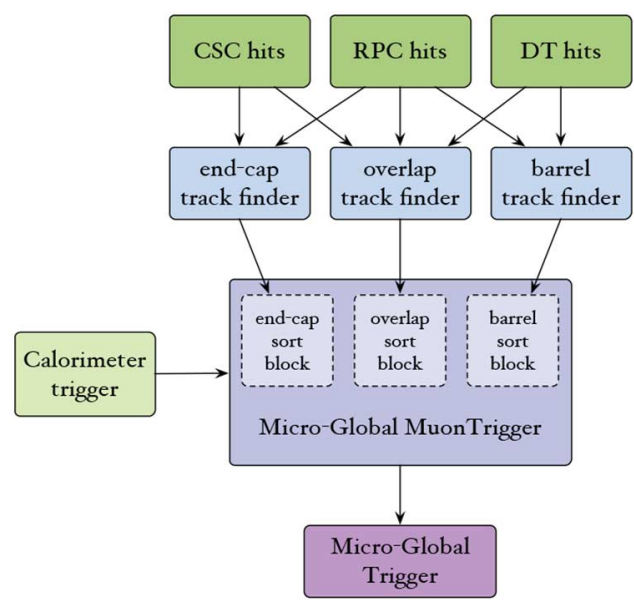

Fig. 3. The upgraded muon trigger chain, starting at the muon detector hits via the track finders to the upgraded GMT that includes the sorting stage.

trigger only information from RPCs and Thin Gap Chambers is used for triggering, while DT and CSC information is only used in later stages of the muon reconstruction. In contrast to CMS, the positional information is not forwarded to the final module taking the trigger decision, the Central Trigger Processor (CTP). Instead, muon multiplicities for six programmable thresholds are sent to the CTP [7]. To allow for algorithms that trigger on topological signatures of multiple objects, a new module is being installed, the Topological Trigger. It will receive both muon and calorimeter information and send topological trigger information, for example spatial distances between objects, to the CTP [8]. Also an approach to confirm muons with signals from the Tile Calorimeter in agreement with minimum ionizing particles is being considered [9].

\section{THE UPGRADED MUON TRIGGER}

In the legacy muon trigger, DT, CSC and RPC hits were handled independently of each other before being merged in the GMT. The upgraded system will aim for a more integrated approach. As mentioned previously the instrumentation in the muon system defines three regions which have different requirements for track finding. Therefore, the upgraded system will feature three track finders, each dedicated to one of these detector regions. The block diagram in Fig. 3 shows the data-flow of the upgraded muon trigger. All track finders will integrate the RPC information [1].

The upgraded GMT ( $\mu \mathrm{GMT})$ will receive muon information directly from the individual processing boards of the track finders. The sorting, currently performed in dedicated boards, will be performed by the $\mu \mathrm{GMT}$ itself which reduces the overall latency by the latency of a serial optical link which includes the (de)serialization steps. The $\mu \mathrm{GMT}$ will receive a total of 108 muon candidates per bunch crossing, 36 candidates per track finder. Muons are transmitted in a 64 bit word, encoding the same information as in the legacy trigger. Table I shows the number of bits assigned for each variable and a comparison with the current system. A rather large portion of the remaining bits is reserved, to allow for additional information to be added if need be. One possible use of the reserved bits would be a unique labeling scheme for the hits to be used in duplicate track removal. Additionally, a few bits are foreseen to identify the associated bunch crossing and technical information for synchronization.

The functionality to tag isolated muons based on an isolation bit received from the calorimeter trigger, which was implemented already in the legacy GMT, was not used during Run-I [10]. The $\mu$ GMT will receive the actual energy values from the calorimeter system to allow a more complex calculation of the isolation variable. These values will be encoded in a linear 5 bit scale where the highest value acts as an overflow bin. For the isolation regions of a radius $R=\sqrt{\Delta \phi^{2}+\Delta \eta^{2}}=0.5$ will be used. These energy sums can be calculated from smaller regions in either the $\mu \mathrm{GMT}$ or the calorimeter trigger. In both cases, to cover the whole muon acceptance the $\mu$ GMT receives up to 1008 energy sums.

\section{PROCESSING BOARD}

The upgraded CMS trigger system will be based on $\mu \mathrm{TCA}$ [11], providing redundant power sources, a high bandwidth backplane and many possibilities for monitoring the overall system-health. The $\mu$ GMT is developed for a Virtex-7 based processing board, the Master Processor 7 (MP7) built by Imperial College. The MP7 is an Advanced Mezzanine Card (AMC) that features a XC7VX690T Xilinx Virtex-7 chip. While the legacy GMT was implemented in ten Virtex-II chips, the new card will provide roughly an increase of a factor of 3 in logic cells and a factor of 7 in memory. The Virtex-7 FPGA on the card features transceivers capable of input and output bandwidth of $13.1 \mathrm{~Gb} / \mathrm{s}$. The MP7 provides $72+72$ optical front-panel links, using Multi-fiber Termination Push-on (MTP) connectors bundling 36 fibres each. A $10 \mathrm{~Gb} / \mathrm{s}$ asynchronous link-protocol will be used both for the incoming and outgoing connections. A more detailed description and specifications of the MP7 may be found elsewhere [12].

\section{FIRMWARE AND ALGORITHMS}

Fig. 4 shows the block diagram of the $\mu$ GMT algorithm logic that will be described in this section. As depicted in the figure, the logic can be split in two major blocks, the isolation assignment and the selection of the best eight muons. The latter consists of a sorting algorithm and cancellation of redundant muons. Data are received on the serial $10 \mathrm{~Gb} / \mathrm{s}$ links with 32 bits per link at $240 \mathrm{MHz}$. The data are deserialized and passed to the algorithm logic as 64 bit muon objects. Pre-processing of the muons is performed at $120 \mathrm{MHz}$ while the main algorithm runs at the LHC clock frequency of $40 \mathrm{MHz}$. The individual algorithm blocks are described in more detail in the following sub-sections. 


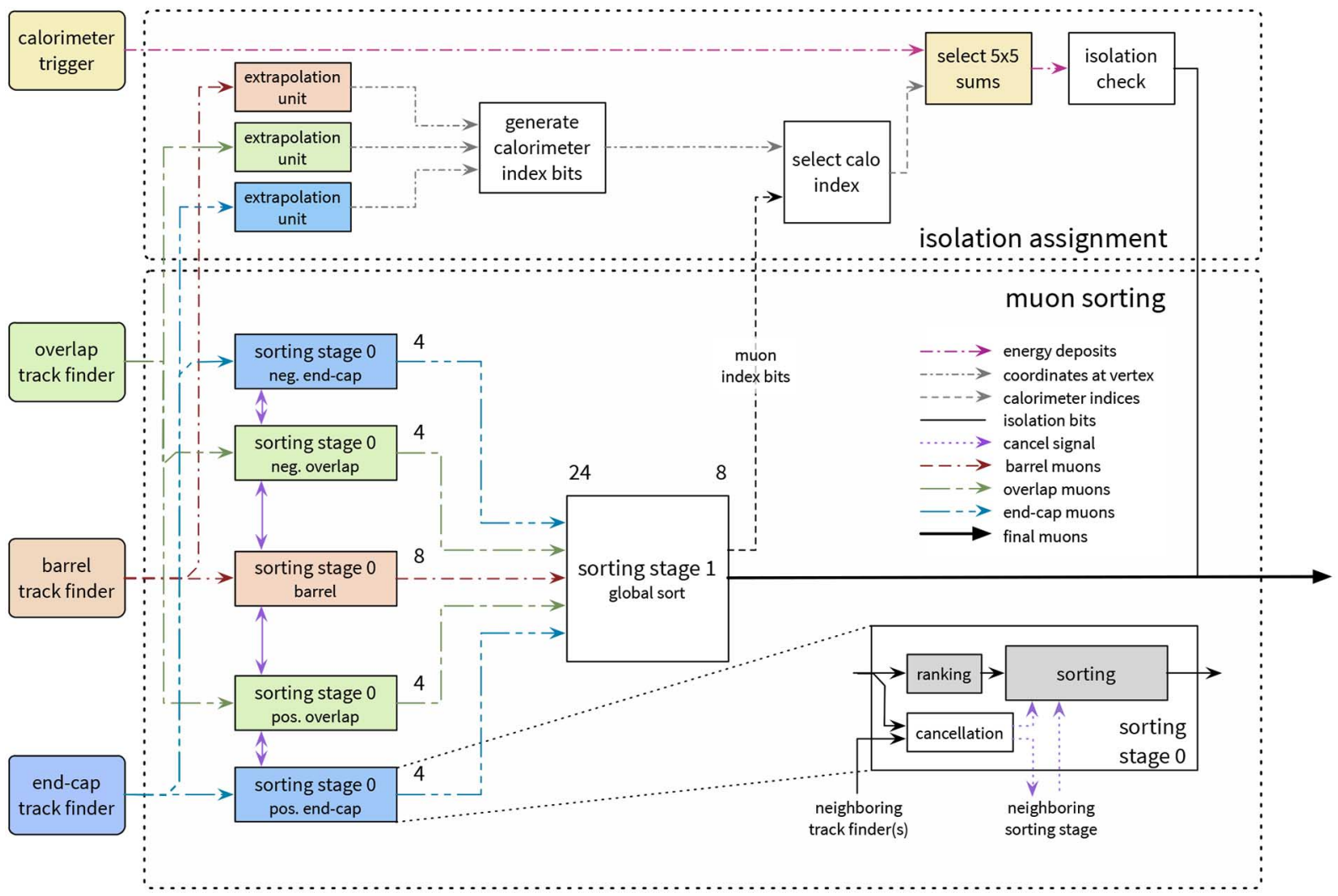

Fig. 4. The upgraded muon trigger algorithm block diagram.

\section{A. Sorting}

To manage the high number of incoming muons, the sorting is split into two stages. In a first stage, sorting stage 0 in Fig. 4, the muons are sorted individually per track finder, splitting the end-cap and overlap track finder candidates into two collections for positive and negative $\eta$ as shown in Fig. 4. The sorting is based on a rank that is calculated from the muon candidate quality and $p_{T}$, as indicated in the enlarged inset at the lower right of Fig. 4. This rank is compared to all other ranks of muons from the same track finder region. For the barrel sorting, the 8 muons of highest rank are forwarded to the second stage. For both overlap and end-cap muons, the 4 best candidates from both negative and positive $\eta$ are used. In the second stage, stage 1 in Fig. 4, the final 8 are selected from the preselected 24. The method is repeated re-using the previously assigned ranks. The rank used for sorting is assigned at $120 \mathrm{MHz}$. This pipelined processing significantly reduces the memory resources needed, as one look-up table can be used for three muons in one bunch crossing.

\section{B. Cancellation}

One important function of the $\mu \mathrm{GMT}$ is to remove muon candidates that were found more than once. The track finding is done in multiple processors and each receives only information from a sub-set of muon chambers, referred to as sectors. The individual processors do not exchange information and muons detected at the boundaries may be found by both. Additionally, due to the geometry of the CMS detector, muons may be measured in two neighboring track finders, overlap and barrel or overlap and end-cap. To avoid double counting of these duplicate muons, a cancellation has to be performed. As depicted in the lower right of Fig. 4, this is done in parallel to the first sorting stage. The cancellation however must be fine-tuned in order not to cancel out signals from real di-muons. This can be done based on the spatial $(\eta, \phi)$ coordinates of the muon, as in the current GMT [2]. Alternatively, unique addresses could be assigned to the candidates that identify the detector elements giving rise to the track elements. The latter approach is used in the sorters for the DT track finder of the current system [10]. Spare bits are reserved for these track-addresses if currently on-going studies should prove that this approach leads to increased performance.

\section{Muon Isolation}

An isolation variable will be calculated in the $\mu \mathrm{GMT}$ to tag muons that have coincidences with large energy deposits in the calorimeter. This tag is then used in the upgraded GT for the final trigger decision. As mentioned previously, isolation is used to distinguish between muons from in-flight decays and those from the actual collision process. It is a widely used technique for background suppression in analyses of fully reconstructed muons [4].

To establish the coincidence with the muon, calorimeter energy deposits in a radius around the muon track are considered. However, especially for muons with low $p_{T}$ the bending due to the magnetic field is of the order of the cone size in which energy deposits are considered. The particles from the hadronization process that carry most of the energy have a much higher momentum than the muon. These particles are therefore 

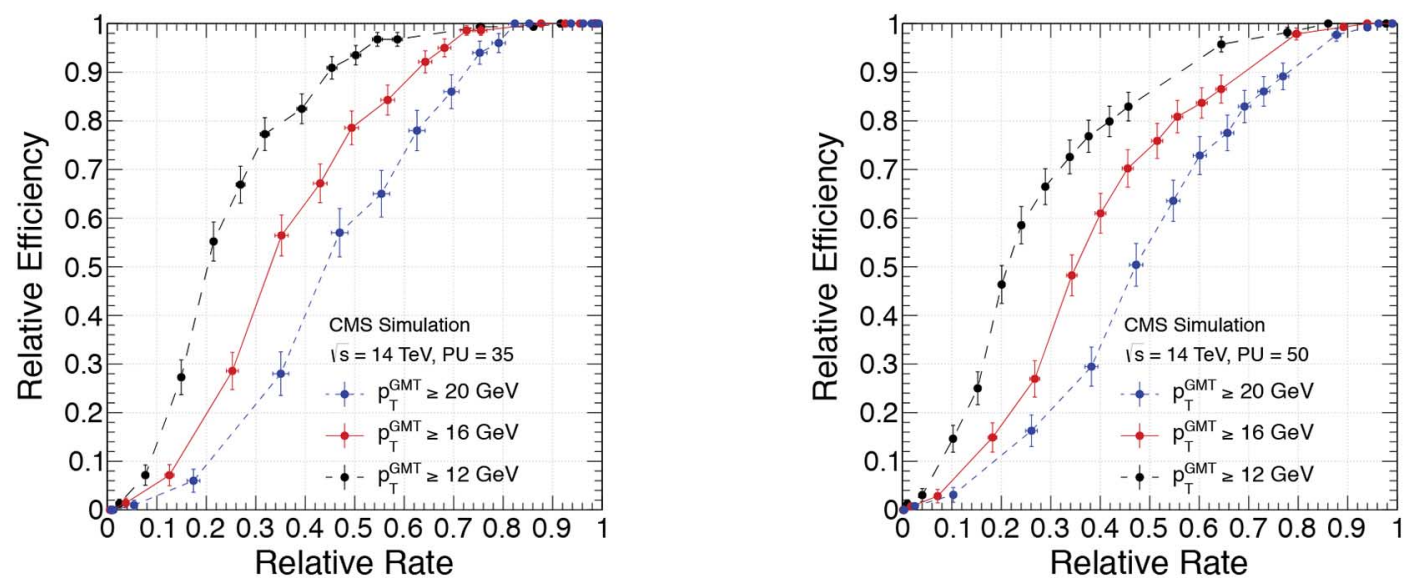

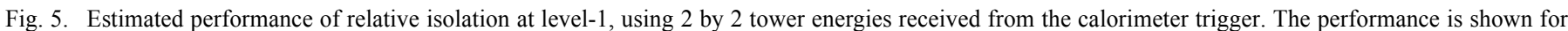

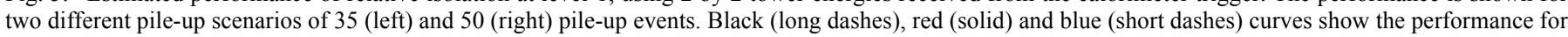
a threshold of $12 \mathrm{GeV}, 16 \mathrm{GeV}$ and $20 \mathrm{GeV}$, respectively [1].

bent much less. Hence, the muons initial $\eta$ and $\phi$ coordinates have to be calculated, the coordinates measured in the muon system are extrapolated to the collision vertex. Look-up tables are defined for different $\eta$-bins that represent $\Delta \phi\left(p_{T}\right)$, the difference between the $\phi$ measured at the vertex and measured in the muon system, as a function of $p_{T}$. In the $\mu$ GMT the extrapolation is performed for all received muons in parallel at $120 \mathrm{MHz}$. The extrapolated $\phi$ and $\eta$ define the center of the calorimeter region considered. The calorimeter is segmented in towers, $\Delta \phi \times \Delta \eta=0.087 \times 0.087$ in the barrel and increasing size $\Delta \eta$ for higher $\eta$. The energy information is only needed within the muon acceptance in CMS $(|\eta|<2.4)$, which reduces the number of required calorimeter towers. Even with the high bandwidth available in the upgraded trigger, the $\mu$ GMT cannot receive calorimeter data at full granularity. Therefore, it will receive regions composed of 2 towers in $\phi$ and 1-2 towers in $\eta$. Alternatively, cones with a radius of 4 towers centered around every second tower could be used. In the first case, the $\mu$ GMT will produce sums of $5 \times 5$ tower regions, in the latter, the cone-energies will be used directly for the isolation. The energy sums that are near the muon tracks of the final eight muons are selected and used to tag the muons both for a relative isolation and absolute isolation. For the relative isolation the energy is divided by the muon $p_{T}$, while for the absolute isolation the energy itself is used. The variables are checked to be smaller than a programmable threshold and the muon is tagged accordingly.

\section{Isolation Performance StUdies}

Studies regarding the previously mentioned improvement of $p_{T}$ assignment within the track finders are currently under way. In this paper we concentrate on the presentation of the muon isolation performance.

Studies were performed on simulated events with a centerof-mass energy of $14 \mathrm{TeV}$ produced for two likely pile-up scenarios of 35 and 50 events per bunch crossing. For this study, it was assumed that the $\mu$ GMT will receive energies of $2 \times 2$ tower regions and calculate the isolation variable as described in Section IV-C. The study was based on the simulation of the legacy muon trigger and the expected isolation performance was evaluated relative to the performance of the GMT without isolation. As a measure of performance, the change in efficiency for signal and background are compared for different isolation thresholds.

To estimate the background efficiency, a Monte Carlo simulation of minimum bias events was used. The only selection criterion was a level-1 trigger muon above a $p_{T}$ threshold of $12 \mathrm{GeV}, 16 \mathrm{GeV}$ or $20 \mathrm{GeV}$. The background efficiency then corresponds to the relative rate $r_{r e l}=N_{i s o} / N_{t o t}$, with $N_{i s o}$ the number of isolated trigger muons and $N_{t o t}$ the total number of trigger muons.

The signal efficiency calculation was based on a $W$ boson production simulation. The same level-1 $p_{T}$ thresholds were required as for the background sample. The reconstructed muons from the leptonic decay of the $W$ boson are selected by performing a matching to the generator muon. The selected reconstructed muons are required to have a $p_{T} 8 \mathrm{GeV}$ higher than the level-1 $p_{T}$ threshold. A spatial match is performed between the reconstructed muon and the GMT muon candidates with $\Delta R$ $=\sqrt{\Delta \phi^{2}+\Delta \eta^{2}}<0.5$. The relative efficiency is calculated $\varepsilon_{r e l}=N_{i s o} / N$, where $N$ is the number of reconstructed muons that can be matched to GMT muon candidates and $N_{i s o}$ is the sub-set of these GMT muon candidates that also fulfill the isolation criterion.

Fig. 5 shows the expected performance for the relative isolation at level-1 comparing relative efficiency to relative rate. Each point corresponds to a certain cut on the isolation variable. The expected performance of relative isolation is shown for two different pile-up scenarios, an average pile-up of 35 (Fig. 5 left hand side) and 50 (Fig. 5 right hand side). In each plot, three lines are shown representing the performance relative to the three different GMT $p_{T}$ thresholds of $12 \mathrm{GeV}, 16 \mathrm{GeV}$ and $20 \mathrm{GeV}$. The change in performance for different $p_{T}$ thresholds is due to the additional $p_{T}$-dependence of the relative isolation. The curves show that high thresholds for the isolation do not reject any signal muons but also do not reduce the rate. Low thresholds can reduce the rate significantly but also decrease the efficiency for the signal. However, the curves show also that for some thresholds the rate can be reduced, while the 
relative efficiency can be kept close to $100 \%$. For example, at a $2 \%$ efficiency loss a reduction of about $10-30 \%$ can be achieved, depending on the pile-up scenario and $p_{T}$ threshold. Thus, the introduction of the isolation offers the possibility to extend the traditional $p_{T}$ thresholds with an isolation criterion to further suppress rate while maintaining a similar efficiency.

Recent studies have shown that the $p_{T}$ resolution, which is expected to be improved by the new track finder algorithms, has a large influence on the isolation performance. With an improved $p_{T}$ resolution the rate reduction can be increased to 20-40\%, depending on the $p_{T}$ threshold.

\section{SUMMARY}

The new running conditions of the LHC in Run-II confront the experiments with new challenges, as the event rate increases significantly. Without changes to the triggering scheme, level-1 trigger thresholds would have to be increased, reducing the efficiency of selecting interesting physics events. This necessitates the upgrade of the level-1 trigger system of CMS. We presented the plans for the upgraded Global Muon Trigger, to be operational from 2016 onwards.

The concept of the legacy system is modified to combine the information from the different muon systems at an earlier stage of the trigger chain. Thereby, a better momentum measurement can be achieved. The previously independent sorting stage will be moved into the $\mu \mathrm{GMT}$. This integrated approach optimizes the latency of the muon trigger path.

For the first time energy measurements from the calorimeter are combined with the muon information in the level-1 trigger scheme. Muon isolation is introduced and will be calculated in the $\mu \mathrm{GMT}$. The studies presented show that the isolation will roughly reduce the rate by $10 \%-30 \%$ at a small efficiency loss.
The rate reduction could be increased if the track finder momentum resolution is improved, which is one of the main goals of the new algorithms for the upgraded muon track finders.

A first version of the algorithm-firmware was implemented and successfully placed in the target Xilinx Virtex-7 chip and is currently being tested.

\section{REFERENCES}

[1] CMS Collaboration, "CMS technical design report for the level-1 trigger upgrade, CERN/LHCC," pp. 2013-011, 2013.

[2] CMS Collaboration, "The CMS experiment at the CERN LHC," 2008, JINST 3 S08004.

[3] R. Frühwirth, M. Regler, R. K. Bock, H. Grote, and D. Notz, Data Analysis Techniques for High-Energy Physics, 2nd ed. Cambridge, U.K.: Cambridge Univ. Press, 2000.

[4] CMS Collaboration, Performance of CMS Muon Reconstruction in PP Collision Events at $\mathrm{sqrt}(\mathrm{s})=7 \mathrm{TeV}, 2012$, JINST 7 P10002.

[5] M. Konecki, The RPC Based Trigger for the CMS Experiment at the LHC, 2014, JINST 9 C07002.

[6] N. M. Nikityuk and V. N. Samoilov, "Review of the trigger systems of the ATLAS and CMS detectors at the LHC," Phys. Part. Nucl., vol. 38, pp. 659-697, 2007.

[7] ATLAS Collaboration, "Performance of the ATLAS muon trigger in 2011 ATLAS-CONF-2012-099," 2012.

[8] S. Artz, B. Bauss, H. Boterenbroodb, V. Bueschera, A. S. Cerqueiraf, and R. Degele et al., The ATLAS Level-1 Muon Topological Trigger Information for Run 2 of the LHC, 2014, ATL-DAQ-PROC-2014-041.

[9] T. Ciodaro, J. M. de Seixas, and A. Cerqueira, "Use of hadronic calorimetry information in the ATLAS level-1 muon trigger," IEEE Trans. Nucl. Sci., vol. 61, no. 2, pp. 1047-1055, Apr. 2014.

[10] CMS Collaboration, "Technical design report volume 1: The trigger systems," pp. 2000-38, 2000, CERN/LHCC.

[11] PCI Industrial Computer Manufacturers Group, Micro telecommunications computing architecture base specification: Micro TCA, PICMG, 2006 [Online]. Available: https://www.picmg.org/wp-content/uploads/ MicroTCA_Short_Form_Sept_2006.pdf

[12] K. Compton, S. Dasu, A. Farmahini-Farahani, S. Fayer, R. Fobes, and R. Frazier et al., The MP7 and CTP-6: Multi-Hundred Gbps Processing Boards for Calorimeter Trigger Upgrades at CMS, 2012, JINSTxg 7 C12024. 